\title{
The impact of virus infections on pneumonia mortality is complex in adults: a prospective multicentre observational study
}

Naoko Katsurada ${ }^{1,2}$, Motoi Suzuki ${ }^{3^{*}}$ (D, Masahiro Aoshima ${ }^{1}$, Makito Yaegashi ${ }^{4}$, Tomoko Ishifuji ${ }^{3}$, Norichika Asoh $^{5}$, Naohisa Hamashige ${ }^{6}$, Masahiko Abe ${ }^{7}$, Koya Ariyoshi $^{3}$, Konosuke Morimoto $^{3}$ and on behalf of the Adult Pneumonia Study Group-Japan

\begin{abstract}
Background: Various viruses are known to be associated with pneumonia. However, the impact of viral infections on adult pneumonia mortality remains unclear. This study aimed to clarify the effect of virus infection on pneumonia mortality among adults stratified by virus type and patient comorbidities.

Methods: This multicentre prospective study enrolled pneumonia patients aged $\geq 15$ years from September 2011 to August 2014. Sputum samples were tested by in-house multiplex polymerase chain reaction assays to identify 13 respiratory viruses. Viral infection status and its effect on in-hospital mortality were examined by age group and comorbidity status.

Results: A total of 2617 patients were enrolled in the study and $77.8 \%$ was aged $\geq 65$ years. 574 (21.9\%) did not have comorbidities, 790 (30.2\%) had chronic respiratory disease, and 1253 (47.9\%) had other comorbidities. Viruses were detected in 605 (23.1\%) patients. Human rhinovirus (9.8\%) was the most frequently identified virus, followed by influenza A (3.9\%) and respiratory syncytial virus (3.9\%). Respiratory syncytial virus was more frequently identified in patients with chronic respiratory disease (4.7\%) than those with other comorbidities (4.2\%) and without comorbidities (2.1\%) ( $p=0.037)$. The frequencies of other viruses were almost identical between the three groups. Virus detection overall was not associated with increased mortality (adjusted risk ratio (ARR) $0.76,95 \% \mathrm{Cl} 0.53-1.09$ ). However, influenza virus A and B were associated with three-fold higher mortality in patients with chronic respiratory disease but not with other comorbidities (ARR 3.38, 95\% CI 1.54-7.42). Intriguingly, paramyxoviruses were associated with dramatically lower mortality in patients with other comorbidities (ARR $0.10,95 \% \mathrm{Cl} 0.01-0.70$ ) but not with chronic respiratory disease. These effects were not affected by age group.
\end{abstract}

Conclusions: The impact of virus infections on pneumonia mortality varies by virus type and comorbidity status in adults.

Keywords: Pneumonia mortality, Chronic respiratory disease, Respiratory syncytial virus, Respiratory virus, Influenza, Paramyxovirus

\footnotetext{
* Correspondence: mosuzuki@nagasaki-u.ac.jp

${ }^{3}$ Department of Clinical Medicine, Institute of Tropical Medicine, Nagasaki

University, 1-12-4 Sakamoto, Nagasaki 852-8523, Japan

Full list of author information is available at the end of the article
}

(c) The Author(s). 2017 Open Access This article is distributed under the terms of the Creative Commons Attribution 4.0 International License (http://creativecommons.org/licenses/by/4.0/), which permits unrestricted use, distribution, and reproduction in any medium, provided you give appropriate credit to the original author(s) and the source, provide a link to the Creative Commons license, and indicate if changes were made. The Creative Commons Public Domain Dedication waiver (http://creativecommons.org/publicdomain/zero/1.0/) applies to the data made available in this article, unless otherwise stated. 


\section{Background}

Pneumonia is the major cause of morbidity and mortality among adults, especially in the elderly. Management of pneumonia is a critical problem in an ageing society like Japan. Streptococcus pneumoniae and Haemophilus influenzae are the leading bacterial causes of adult pneumonia, while viruses also play important roles in disease development. Recent advances in molecular diagnostic techniques have enabled us to detect multiple viruses simultaneously [1]. Studies have shown that viral infection is common in pneumonia patients [2, 3]. According to a recent systematic review and meta-analysis, viruses were detected in $24.5 \%$ of respiratory samples from community-acquired pneumonia (CAP) patients [4].

Various viruses are known to be associated with respiratory infections, including pneumonia. According to the systematic review, influenza is the most commonly detected virus in CAP, followed by human rhinovirus (HRV), respiratory syncytial virus (RSV), and human coronavirus (HCoV) [4]. In addition to these endemic respiratory viruses, emerging respiratory viruses, such as severe acute respiratory syndrome coronavirus, Middle East respiratory syndrome coronavirus, and avian influenza, are posing a particularly serious threat to global health security [5]. Studies have suggested that these emerging viral infections are associated with an increased risk of severe conditions and mortality among pneumonia patients $[5,6]$. It must be noted that pneumonia mortality varies substantially according to patient characteristics, such as comorbidities, aspiration risk factors, and physical functional status $[7,8]$. To establish effective control measures, high-priority viruses and patient groups must be identified. However, the prevalence of viruses in adult pneumonia and their virus-specific effects on clinical outcome remain largely unknown. To the best of our knowledge, no large-scale study has investigated the different effects of viruses on pneumonia mortality by patient characteristics.

We conducted this prospective multicentre study to determine the distribution of viruses associated with pneumonia in adults and to establish their virus-specific effects on pneumonia mortality stratified by age group and comorbidity status.

\section{Methods}

\section{Study design, patient enrolment, and data collection}

The Adult Pneumonia Study Group-Japan (APSG-J) conducted multicentre prospective hospital-based surveillance for community-onset pneumonia at four community-based hospitals in Japan. In our previous paper, the burden and aetiology of adult pneumonia were reported based on the data and clinical samples collected during the 1st phase of the study (September 2011 to January 2013) [9]. The current study included all data and samples collected during the whole study period (September 2011 to August 2014). Details of the study settings and enrolment criteria were described previously [9]. In brief, all outpatients and inpatients were screened by hospital physicians, and eligible patients were identified using a standardized case definition: patients aged $\geq 15$ years with respiratory symptoms compatible with pneumonia and new infiltrative shadows on chest Xrays or computed tomography scans. Clinical information was collected from patients and medical charts using a standardized data collection form.

\section{Microbiological test}

Sputum, blood, and urine samples were collected at the time of diagnosis. Gram staining, sputum culture, and blood culture were performed on site. Sputum samples were further tested by in-house multiplex polymerase chain reaction (PCR) assays to identify viral and bacterial pathogens at the Institute of Tropical Medicine, Nagasaki University. Thirteen viral pathogens (influenza A virus, influenza B virus, RSV, human metapneumovirus [hMPV], human parainfluenza virus [HPIV] type 1-4, HRV, $\mathrm{HCoV}$ 229E/OC43, human adenovirus [HAdV], and human bocavirus $[\mathrm{HBoV}])$ and six bacterial pathogens (Streptococcus pneumoniae, Haemophilus influenzae, Moraxella Catarrhalis, Mycoplasma pneumoniae, Chlamydophila pneumoniae, and Legionella pneumophila) were tested using multiplex PCR assays. Details about the primers and PCR methods used have been described previously [10, 11]. Urinary antigen testing was performed for the detection of S. pneumoniae and L. pneumophila (Binax NOW Streptococcus Pneumoniae, Binax NOW Legionella; Alere Inc., Waltham, MA, USA).

\section{Definitions of variables}

Diagnosis of viral infection was made according to PCR results. Bacterial infection was diagnosed when any of the following criteria were fulfilled: 1) culture yielded pathogenic bacteria from microscopically purulent sputum samples (i.e., Geckler's classification groups 4 and 5) or normally sterile site samples; 2) PCR assays were positive for bacterial DNA in microscopically purulent sputum samples; or 3) urinary antigen tests showed a positive result.

Patients were categorized into four age groups: 15-64 years, 65-74 years, 75-84 years, and $\geq 85$ years. Patients' disability status was evaluated using the Eastern Cooperative Oncology Group Performance Status (PS) score [12]. Pneumonia severity was assessed using the CURB65 scoring system [13]. To estimate the effect on pneumonia mortality, viruses were categorized into four groups: 1) HRV; 2) influenza A and B viruses; 3) paramyxoviruses (RSV, hMPV, and PIV type 1-4); and 4) other viruses (HAdV, $\mathrm{HBoV}$, and $\mathrm{HCoV}$ ). 
We divided patients into three groups according to comorbidity status: 1) patients without comorbidity; 2) patients with chronic respiratory disease; and 3) patients with comorbidities other than chronic respiratory disease (i.e., other comorbidities). Chronic respiratory disease included bronchial asthma, chronic obstructive pulmonary disease (COPD), interstitial pneumonia, pneumoconiosis, and bronchiectasis. Other comorbidities included diabetes mellitus, cerebrovascular disease, dementia, neuromuscular disease, cardiac failure, ischaemic heart disease, collagen disease, malignancy, renal disease, and liver disease. Patients were considered to have aspiration risk factors when they had any of the following factors: episodes of aspiration, the presence of dysphagia, consciousness disturbances, neuromuscular diseases, cerebrovascular diseases, tube feeding, and bedridden status [14].

The in-hospital death was defined as any death occurred during the hospitalization. During the first year of study, we followed up our patients after the enrolment and confirmed that no outpatient had died within 30 days of enrolment. We therefore considered the in-hospital death as a good marker of short-term mortality in pneumonia patients regardless of their hospitalization status.

\section{Statistical analysis}

Patients were categorized according to their comorbidity status (i.e., patients without comorbidity, with chronic respiratory disease, or with other comorbidities) and compared using chi-squared tests. Viral and bacterial infection status were compared by age group and comorbidity status using chi-squared tests, Fisher's exact tests, and chi-squared tests for trend. In-hospital mortality rates were calculated by viral and bacterial infection status and compared with those of the virus-negative group. The effects of viral infection on in-hospital mortality were expressed as risk ratios with 95\% confidence intervals $(\mathrm{CI})$ and estimated using Poisson regression models with robust standard errors. Age, study site, comorbidity status, duration of symptoms, month of diagnosis, antibiotic use, and presence of bacteria were considered potential confounders based on prior knowledge and were included in the multiple regression models. For patients whose onset of symptoms were unknown $(<5 \%)$, we coded those missing values as "unknown" and included all patients in our analysis. The data were analysed using STATA version 13 (STATA Corp., College Station, TX, USA).

\section{Ethics}

This study was approved by the institutional review boards (IRBs) of the Institute of Tropical Medicine, Nagasaki University, Ebetsu City Hospital, Kameda Medical Center, Chikamori Hospital, and Juzenkai Hospital. The requirement for obtaining written consent from all participants was waived by all IRBs because of the study's observational nature without any deviation from the current medical practice. Anonymized data were used for the analyses.

\section{Results}

\section{Clinical characteristics}

During the study period, 3816 patients were enrolled in the study. Of these, 346 were excluded because of refusal to participate in the study $(n=48)$, absence of pulmonary infiltrates $(n=163)$, and non-pneumonia diagnosis $(n=135)$. After excluding $853(22.3 \%)$ patients whose sputum samples for PCR assays were unavailable, 2617 patients were eligible for analysis (Fig. 1).

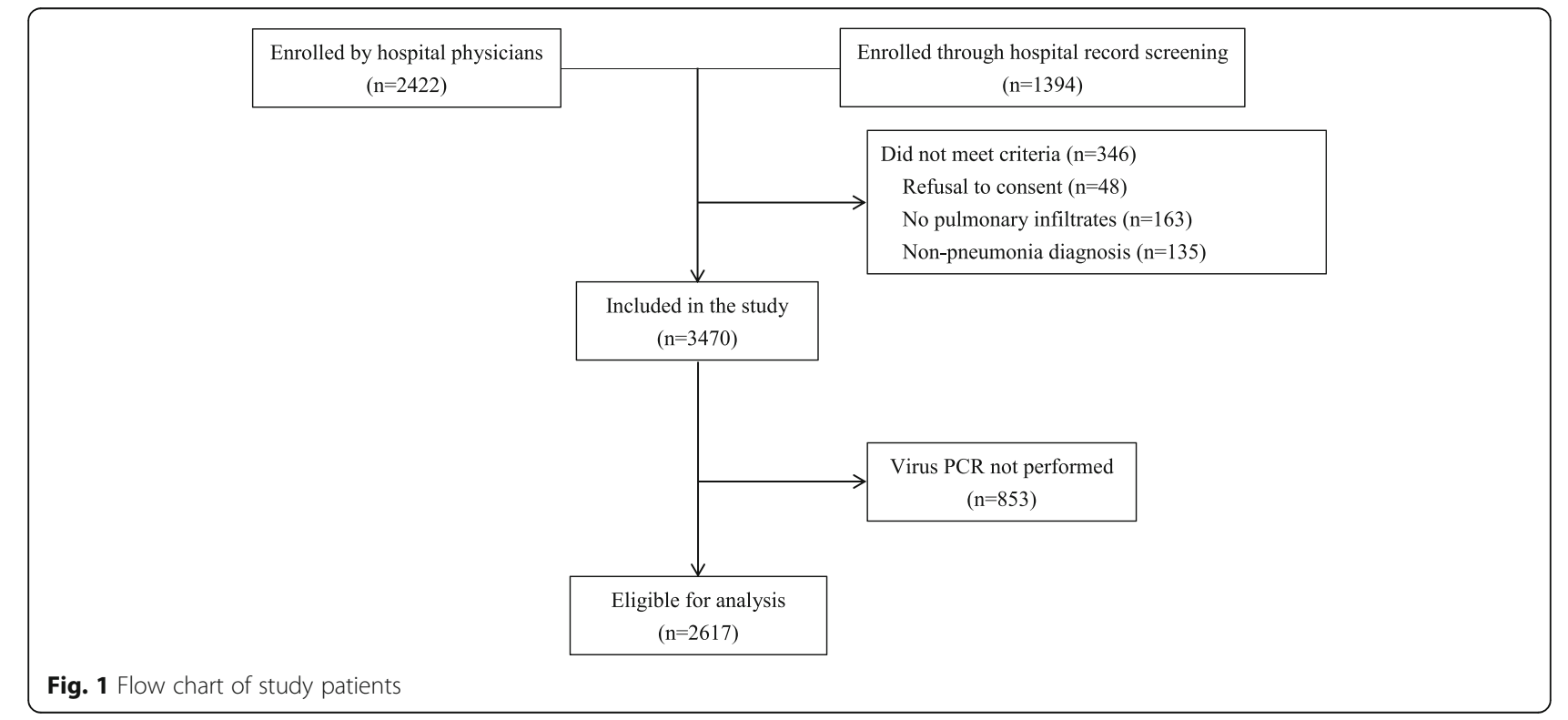


Table 1 shows the clinical characteristics of pneumonia patients by comorbidity status. Approximately $60 \%$ of patients were male, and the median age was 73.8 years (interquartile range, 66 to 85 years). The proportions of patients aged $\geq 65$ years and $\geq 85$ years were 77.8 and $28.4 \%$, respectively; $46.7 \%$ of 2545 patients had aspiration risk factors. Of all patients, 574 (21.9\%) did not have comorbidities, 790 (30.2\%) had chronic respiratory disease, and 1253 (47.9\%) had other comorbidities. Patients with comorbidities were more likely to be male, older, more frequently required hospitalization, more frequently developed severe disease, more frequently had aspiration risk factors, had higher PS scores, and visited the hospital earlier than those without comorbidities. The proportion of patients with aspiration risk factors was particularly high (65.5\%) among patients with other comorbidities. Multiple symptoms were most frequently observed in patients with chronic respiratory disease (70.1\%), followed by those without comorbidities (67.9\%) and those with other comorbidities (57.5\%).

\section{Detection of viral and bacterial pathogens}

In total, $605(23.1 \%)$ patients tested positive for at least one virus (Table 2). HRV was the most common virus identified $(n=256$ [9.8\%]), followed by influenza A $(n=101$ [3.9\%]) and RSV $(n=101$ [3.9\%]). Two or more viruses were detected in 31 patients (1.2\%). The most frequent combinations of viruses were HRV plus influenza A $(n=7)$, followed by HRV plus RSV $(n=4)$, and HRV plus hMPV $(n=4)$. Three viruses (HRV, hMPV, and PIV type 3 ) were detected in one patient. Bacterial pathogens were detected in 992 (37.9\%) patients, and both viral and bacterial pathogens were detected in 246 (9.4\%) patients (i.e., viral-bacterial co-infection).

Viral and bacterial infection status were compared by age group (Table 2) and comorbidity status (Table 3 ). The proportion of overall virus-positive pneumonia did not differ by age group. RSV was more frequently identified in older age groups, while $\mathrm{HCoV}$ was more frequently identified in younger age groups. The proportion of influenza-

Table 1 Characteristics of enrolled pneumonia patients

\begin{tabular}{|c|c|c|c|c|c|}
\hline & $\begin{array}{l}\text { Total } \\
N=2617\end{array}$ & $\begin{array}{l}\text { No comorbidity } \\
N=574\end{array}$ & $\begin{array}{l}\text { Chronic respiratory disease } \\
N=790\end{array}$ & $\begin{array}{l}\text { Other comorbidities }^{a} \\
N=1253\end{array}$ & $P$ valuet \\
\hline & N (\%) & $\mathrm{N}(\%)$ & N (\%) & N (\%) & \\
\hline Male sex & $1591(60.8)$ & $295(51.4)$ & $540(68.4)$ & $756(60.3)$ & $<0.001$ \\
\hline \multicolumn{6}{|l|}{ Age group } \\
\hline $15-64$ & $580(22.2)$ & $268(46.7)$ & $121(15.3)$ & $191(15.2)$ & \multirow[t]{4}{*}{$<0.001$} \\
\hline $65-74$ & $447(17.1)$ & $91(15.9)$ & $141(17.9)$ & $215(17.2)$ & \\
\hline $75-84$ & $848(32.4)$ & $126(22.0)$ & $321(40.6)$ & $401(32.0)$ & \\
\hline $85+$ & $742(28.4)$ & $89(15.5)$ & $207(26.2)$ & $446(35.6)$ & \\
\hline Admission & 2007 (76.7) & $305(53.1)$ & $597(75.6)$ & $1105(88.2)$ & $<0.001$ \\
\hline Performance status $\geq 3, n=1307$ & $310(23.7)$ & $37(11.1)$ & $56(13.9)$ & $217(37.9)$ & $<0.001$ \\
\hline Severe disease (CURB65 $\geq 4$ ), $n=2100$ & $154(7.3)$ & $19(4.5)$ & $41(6.7)$ & $94(8.9)$ & 0.010 \\
\hline Duration of symptoms ${ }^{b}>3$ days, $n=2507$ & $874(34.9)$ & $256(47.8)$ & $251(33.3)$ & $367(30.2)$ & $<0.001$ \\
\hline No. of symptoms $\geq 3$ & $1664(63.6)$ & $390(67.9)$ & $554(70.1)$ & $720(57.5)$ & $<0.001$ \\
\hline Aspiration risk, $n=2545$ & $1189(46.7)$ & $103(18.4)$ & $293(37.8)$ & $793(65.5)$ & $<0.001$ \\
\hline \multicolumn{6}{|l|}{ Period } \\
\hline Spring (January to March) & $645(24.7)$ & $129(22.5)$ & $195(24.7)$ & $321(25.6)$ & \multirow[t]{4}{*}{0.092} \\
\hline Summer (April to June) & $572(21.9)$ & $147(25.6)$ & $161(20.4)$ & $264(21.1)$ & \\
\hline Autumn (July to September) & $641(24.5)$ & $151(26.3)$ & $189(23.9)$ & $301(24.0)$ & \\
\hline Winter (October to December) & $759(29.0)$ & $147(25.6)$ & $245(31.0)$ & $367(29.3)$ & \\
\hline \multicolumn{6}{|l|}{ Study site } \\
\hline Ebetsu & $326(12.4)$ & 79 (13.8) & $123(15.6)$ & $124(9.9)$ & \multirow[t]{4}{*}{$<0.001$} \\
\hline Kamogawa & 1379 (52.7) & $348(60.6)$ & $416(52.7)$ & $615(49.1)$ & \\
\hline Kochi & $600(22.9)$ & $96(16.7)$ & $153(19.4)$ & $351(28.0)$ & \\
\hline Nagasaki & $312(11.9)$ & $51(8.9)$ & $98(12.4)$ & $163(13.0)$ & \\
\hline
\end{tabular}

†Chi-square tests were performed to compare three groups

${ }^{a}$ Other comorbidities include diabetes mellitus, cerebrovascular disease, dementia, neuromuscular disease, cardiac failure, ischaemic heart disease, collagen disease, malignancy, renal disease, and liver disease

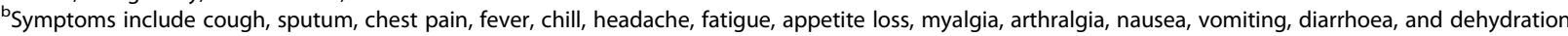


Table 2 Viral and bacterial infection status among pneumonia patients by age group

\begin{tabular}{|c|c|c|c|c|c|c|}
\hline & Total & $15-64 y$ & $65-74 y$ & $75-84$ y & $85 y+$ & $P$ value* \\
\hline Viruses & $N=2617$ & $N=580$ & $N=447$ & $N=848$ & $N=742$ & \\
\hline HRV & $256(9.8)$ & $70(12.1)$ & $42(9.4)$ & $69(8.1)$ & $75(10.1)$ & 0.185 \\
\hline $\operatorname{lnf} A$ & $101(3.9)$ & $22(3.8)$ & $13(2.9)$ & $32(3.8)$ & $34(4.6)$ & 0.343 \\
\hline RSV & $101(3.9)$ & $12(2.1)$ & $20(4.5)$ & $32(3.8)$ & $37(5.0)$ & 0.016 \\
\hline PIV 3 & $49(1.9)$ & $14(2.4)$ & $7(1.6)$ & $17(2.0)$ & $11(1.5)$ & 0.311 \\
\hline HMPV & $45(1.7)$ & $4(0.7)$ & $13(2.9)$ & $14(1.7)$ & $14(1.9)$ & 0.286 \\
\hline PIV 1 & $26(2.0)$ & $5(0.9)$ & $3(0.7)$ & $10(1.2)$ & $8(1.1)$ & 0.523 \\
\hline $\operatorname{lnf} B$ & $24(0.9)$ & $6(1.0)$ & $4(0.9)$ & $3(0.4)$ & $11(1.5)$ & 0.588 \\
\hline PIV 2 & $14(0.5)$ & $4(0.7)$ & $3(0.7)$ & $3(0.4)$ & $4(0.5)$ & 0.567 \\
\hline HCoV (229E/OC43) & $13(0.5)$ & $6(1.0)$ & $3(0.7)$ & $2(0.2)$ & $2(0.3)$ & 0.029 \\
\hline HAdV & $6(0.2)$ & $1(0.2)$ & $1(0.2)$ & $4(0.5)$ & $0(0.0)$ & 0.708 \\
\hline HBoV & $2(0.1)$ & $1(0.2)$ & $0(0.0)$ & $1(0.1)$ & $0(0.0)$ & 0.394 \\
\hline PIV 4 & $0(0.0)$ & $0(0.0)$ & $0(0.0)$ & $0(0.0)$ & $0(0.0)$ & NA \\
\hline Any viruses & $605(23.1)$ & $137(23.6)$ & $102(22.8)$ & $180(21.2)$ & $186(25.1)$ & 0.677 \\
\hline$\geq 2$ viruses & $31(1.2)$ & $8(1.4)$ & $7(1.6)$ & $6(0.7)$ & $10(1.3)$ & 0.654 \\
\hline Any bacterial pathogens & $992(37.9)$ & $265(45.7)$ & $194(43.4)$ & $288(34.0)$ & $245(33.0)$ & $<0.001$ \\
\hline Viral-bacterial co-infection & $246(9.4)$ & $66(11.4)$ & $46(10.3)$ & $69(8.1)$ & $65(8.8)$ & 0.056 \\
\hline Any viral and bacterial pathogens & $1351(51.6)$ & $336(57.9)$ & $250(55.9)$ & $399(47.1)$ & $366(49.3)$ & $<0.001$ \\
\hline
\end{tabular}

HRV human rhinovirus, InfA influenza A virus, RSV respiratory syncytial virus, PIV1-4 human parainfluenza virus type 1-4, HMPV human metapneumovirus, InfB influenza B virus, HCoV human coronavirus (229E/OC43), HAdV human adenovirus, HBoV human bocavirus

${ }^{*}$ Chi-square tests for trend were performed

Table 3 Viral and bacterial infection status among pneumonia patients by comorbidity status

\begin{tabular}{|c|c|c|c|c|}
\hline & No comorbidities & Chronic respiratory disease & Other comorbidities ${ }^{a}$ & $P$ valuet \\
\hline Viruses & $N=574$ & $N=790$ & $N=1253$ & \\
\hline HRV & $57(9.9)$ & $87(11.0)$ & $112(8.9)$ & 0.304 \\
\hline $\operatorname{lnf} A$ & $21(3.7)$ & $29(3.7)$ & $51(4.1)$ & 0.866 \\
\hline RSV & $12(2.1)$ & $37(4.7)$ & $52(4.2)$ & 0.037 \\
\hline PIV 3 & $12(2.1)$ & $11(1.4)$ & $26(2.1)$ & 0.492 \\
\hline HMPV & $6(1.1)$ & $13(1.7)$ & $26(2.1)$ & 0.286 \\
\hline PIV 1 & $4(0.7)$ & $10(1.3)$ & $12(1.0)$ & 0.598 \\
\hline $\operatorname{lnf} B$ & $5(0.9)$ & $5(0.6)$ & $14(1.1)$ & 0.596 \\
\hline PIV 2 & $3(0.5)$ & $4(0.5)$ & $7(0.6)$ & 1.000 \\
\hline HCoV (229E/OC43) & $3(0.5)$ & $5(0.6)$ & $5(0.4)$ & 0.767 \\
\hline HAdV & $2(0.4)$ & $1(0.1)$ & $3(0.2)$ & 0.764 \\
\hline $\mathrm{HBOV}$ & $0(0.0)$ & $0(0.0)$ & $2(0.2)$ & 0.711 \\
\hline PIV 4 & $0(0.0)$ & $0(0.0)$ & $0(0.0)$ & NA \\
\hline Any viruses & $118(20.6)$ & $196(24.8)$ & $291(23.2)$ & 0.183 \\
\hline$\geq 2$ viruses & $7(1.2)$ & $6(0.8)$ & $18(1.4)$ & 0.386 \\
\hline Any bacterial pathogens & $233(40.6)$ & $315(39.9)$ & $444(35.4)$ & 0.043 \\
\hline Viral-bacterial co-infection & $54(9.4)$ & $88(11.1)$ & $104(8.3)$ & 0.101 \\
\hline Any viral and bacterial pathogens & $297(51.7)$ & $423(53.5)$ & $631(50.4)$ & 0.373 \\
\hline
\end{tabular}

HRV human rhinovirus, InfA influenza A virus, RSV respiratory syncytial virus, PIV1-4 human parainfluenza virus type 1-4, $H M P V$ human metapneumovirus, InfB influenza B virus, HCoV human coronavirus (229E/OC43), HAdV human adenovirus, HBoV human bocavirus

+ Chi-square tests or Fisher's exact tests were performed to compare the three groups

${ }^{a}$ Other comorbidities include diabetes mellitus, cerebrovascular disease, dementia, neuromuscular disease, cardiac failure, ischaemic heart disease, collagen

disease, malignancy, renal disease, and liver disease 
positive pneumonia was similar across all age groups. Bacterial pathogens were more frequently identified in younger patients. For patients' comorbidity status, RSV was most frequently identified in patients with chronic respiratory disease $(4.7 \%)$, followed by those with other comorbidities (4.2\%) and without comorbidities (2.1\%) $(p=$ 0.037); the frequencies of other viruses were almost identical between the three groups (Table 3). Bacterial pathogens were more frequently identified in patients without comorbidities than in those with comorbidities.

We explored symptoms of patients with each respiratory virus groups (Additional file 1: Table S1). The proportion of patients with multiple symptoms (i.e., the number of symptoms $\geq 3$ ) was higher in patients with paramyxovirus infection than those without viral infection $(75.0 \%$ vs $61.3 \%, p<0.001)$. In the group of patients with aspiration risk factors, those with paramyxovirus were more likely to have a cough than patients without virus $(71.1 \%$ vs $46.2 \%, p<0.001)$.

\section{In-hospital mortality of pneumonia and virus detection}

Among 2617 patients, 193 patients died before discharge, with an overall in-hospital mortality of $7.4 \%$. The mortalities among virus-positive and -negative groups were 5.8 and $7.9 \%$, respectively, and the overall effect (adjusted risk ratio $[\mathrm{ARR}])$ of viruses on mortality was 0.76 (95\% CI $0.53-1.09, p=0.140$ ). Intriguingly, when the effect of specific virus type was analysed, paramyxoviruses, including RSV, hMPV, and PIV type 1-4, were associated with a dramatically lower mortality (ARR $0.29,95 \%$ CI 0.12-0.71, $p=0.007$ ). Among 212 paramyxovirus-positive pneumonia, five died: three were RSV-positive, one was HMPVpositive, and one was HMPV-positive at the enrolment. RSV alone was also associated with a lower mortality (ARR 0.48, 95\% CI 0.18-1.29, $p=0.146$ ), but the association did not reach a statistically significant level. None of the other virus types were associated with mortality.

The virus type-specific effects were further investigated after patients were stratified by age group and comorbidity status (Tables 4 and 5). Similar effects of viruses were seen across all age groups. However, influenza virus A and B were strongly associated with higher mortality in patients with chronic respiratory disease (ARR 3.38, 95\% CI 1.547.42, $p=0.002$ ), while no influenza-related death was observed in those without comorbidity. Intriguingly, paramyxoviruses were associated with markedly lower mortality in patients with other comorbidities (ARR 0.10, 95\% CI $0.01-0.70, p=0.020$ ), but this association was not observed in other groups. HRV was not associated with mortality in the three groups. Virus only, bacteria only, and both virusand bacteria-positive pneumonia demonstrated higher mortality than virus- and bacteria-negative pneumonia in patients with chronic respiratory disease, but these associations did not reach statistically significant levels.
We explored the association between viruses and inhospital mortality in patients with aspiration risk factors (Additional file 1: Table S2). Paramyxovirus was the only virus type significantly associated with reduced mortality in this category of patients (ARR 0.28, 95\% CI 0.09-0.85, $p=0.024)$. Influenza $\mathrm{A}$ and $\mathrm{B}$ were not associated with mortality (ARR 1.78, 95\% CI 0.92-3.47, $p=0.089$ ). The mortality was higher among patients with cough than those without cough $(11.7 \%$ vs $4.9 \%, p<0.001)$.

\section{Discussion}

In this multicentre prospective study, $23.1 \%$ of adult pneumonias were associated with viruses. HRV was the leading virus identified, followed by influenza $\mathrm{A}$ and RSV. This pattern was almost identical across all age groups. Influenza was strongly associated with higher mortality in patients with chronic respiratory disease but not in other groups. Paramyxoviruses, including RSV, hMPV, and PIV type 1-4, were associated with improved survival in patients with other comorbidities, especially in those with aspiration risk factors. To the best of our knowledge, this study is the first to systematically investigate virus-specific effects on pneumonia mortality by age group and comorbidity status among adults.

Viruses are frequently observed in pneumonia patients. According to previous studies, viruses were positive in $23,34,30$ and $28 \%$ of CAP patents in the US [2], Norway [3], UK [15], and China [16], respectively, and $H R V$, influenza $A$, and RSV were the leading viruses identified; these findings were confirmed in our study. However, the role of viruses in pneumonia development and progression has not been fully established. A systematic review showed that the risk of death was higher in patients with viral infection, although the association did not reach a statistically significant level (odds ratio 1.3, 95\% CI 0.8-2.2) [4]. The major limitation of previous studies is that all viruses and patient groups were pooled, which may have overlooked their intergroup differences. In fact, in the current study, viruses overall were not associated with increased mortality among all pneumonia patients (ARR 0.76, 95\% CI 0.53-1.09), but the effects were different by viruses and patient characteristics.

Influenza increased pneumonia mortality by 3.4 -fold (95\% CI 1.54-7.42) in our patients with chronic respiratory disease but did not change the mortality in other patients. Although influenza is known to be an important cause of pneumonia and death, only a few studies have formally compared the mortality of influenza pneumonia with that of non-influenza pneumonia, and the findings have been inconsistent $[17,18]$. On the other hand, previous studies have demonstrated that chronic respiratory disease increases the risk of severe outcome among influenza patients $[19,20]$. Bronchial epithelial cells of COPD are susceptible to replication of influenza virus because of their 


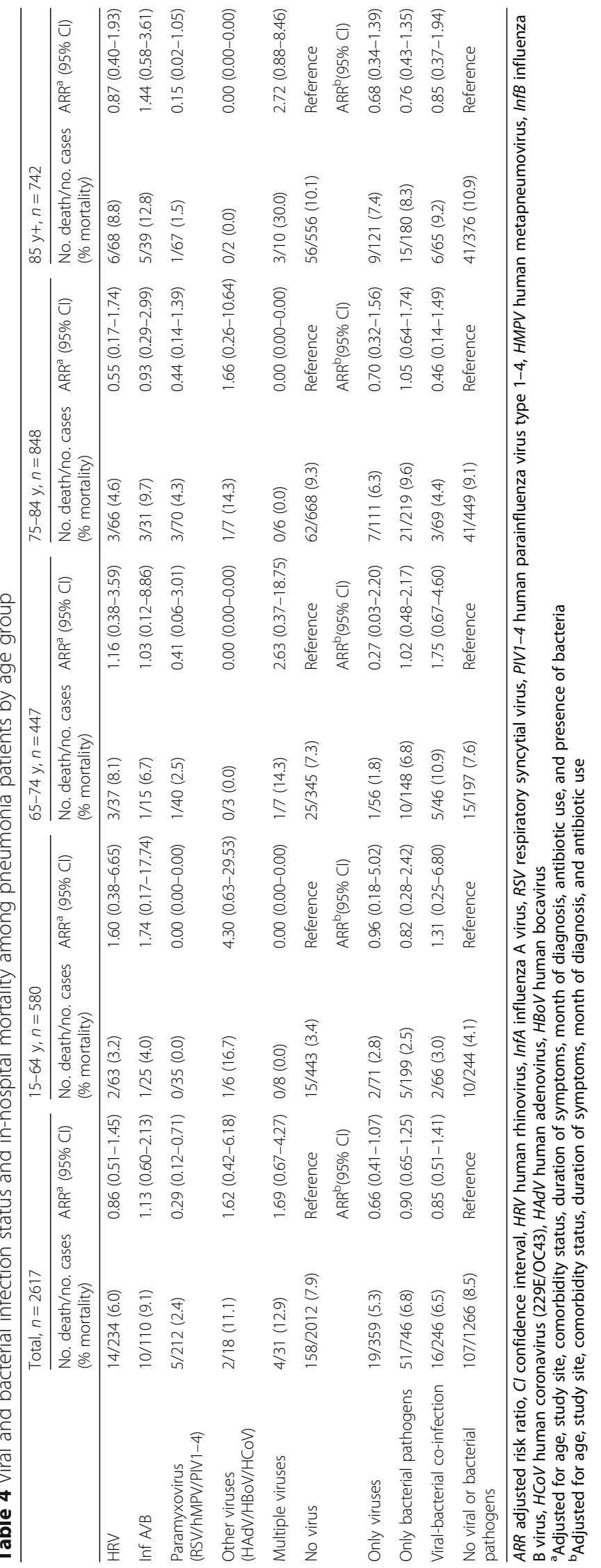




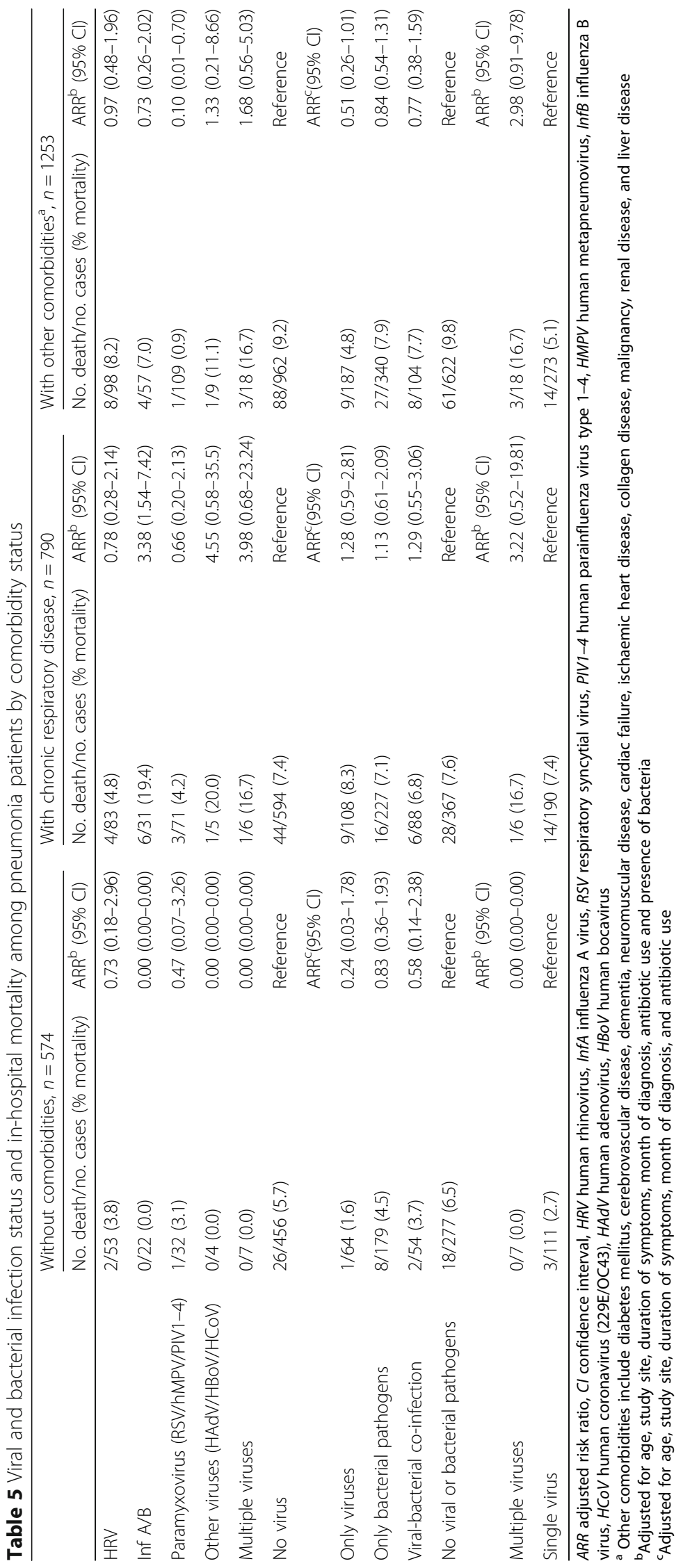


impaired antiviral immunity [21]; thus, the effect of influenza on disease progression may be stronger in patients with this condition. According to the Cochrane review, influenza vaccination reduces exacerbations in patients with COPD [22]. Seasonal influenza vaccination campaigns must therefore pay special attention to this patient group.

Interestingly, paramyxoviruses including RSV were associated with improved survival in our patients with other comorbidities. Inconsistent findings have been reported about the effect of paramyxoviruses on pneumonia severity. A multinational study showed that older patients who had been infected with RSV were more likely to be hospitalized than those with other respiratory viruses [23], while a study conducted in the US demonstrated that patients with RSV infection were less frequently hospitalized than those with influenza infection [24]. A retrospective cohort study conducted in Hong Kong showed that the 30-day and 60-day mortality rates were similar between adult patients hospitalized with RSV and those with seasonal influenza [25]. These inconsistent findings suggest that the effects of paramyxovirus infection substantially vary by patients' conditions. In fact, in the current study, compared with virus-negative pneumonia, the mortality of paramyxovirus-associated pneumonia was substantially lower among patients with other comorbidities but this finding was not observed among patients without comorbidities and patients with chronic respiratory disease. The low mortality of paramyxovirus-associated pneumonia in this groups may be associated with its high prevalence of multiple symptoms. In our study, the proportion of patients with multiple symptoms (i.e., the number of symptoms $\geq 3$ ) was higher in patients with paramyxovirus infection than those without viral infection. Patients with paramyxovirus-associated pneumonia are more likely to develop symptoms and are probably more likely to visit hospitals, and this benefit may be observed in patients with comorbidities. In the group of patients with aspiration risk factors, those with paramyxovirus-associated pneumonia were more likely to have a cough than patients without virus $(71.1 \%$ vs $46.2 \%, p<0.001)$, and the mortality was higher among patients with cough than those without cough $(11.7 \%$ vs $4.9 \%, p<0.001)$. The low mortality of paramyxovirus-associated pneumonia in patients with aspiration risk factors also suggests that these viruses may stimulate the cough reflex and improve patients' survival; however, our study does not provide conclusive evidence. Further studies are needed to unveil the mechanisms of potential benefits of paramyxovirus infection on pneumonia mortality.

In our study, multiple viruses were identified in 5.1\% of virus-associated pneumonia and were associated with higher mortality than single viral infection in patients with chronic respiratory disease and other comorbidities.
The association between multiple viral infections and pneumonia mortality remains uncertain [26]. Systematic reviews have shown that multiple viral infections in patients with respiratory disease are not associated with disease severity [27, 28]; however, the majority of previous studies included young children but not adults. The effect of multiple viruses on disease progression may be different in children and adults. Consistent with previous studies $[3,29,30]$, half of the viral pneumonia patients were co-infected with bacterial pathogens. A systematic review showed that viral-bacterial co-infection increased mortality [4]; however, this association was not observed in our study. Viral infections of the lower respiratory tract may increase the risk of secondary bacterial infection, which may increase the risk of pneumonia and mortality [26]. However, sputum samples were collected once in our study; thus, we were unable to determine the time course of viral and bacterial infections. A cohort study with sequential respiratory sampling may be a preferable design to establish a causal association between viral-bacterial co-infection and pneumonia mortality.

Advances in molecular diagnostic techniques have enabled virus detection in clinical settings. Our findings raise the question of whether all pneumonia patients should be tested for viruses. The increased mortality of influenzaassociated pneumonia in patients with chronic respiratory disease suggests the importance of early diagnosis of influenza and initiation of antivirals for this patient group. On the other hand, no substantial increase of mortality was found for other viruses. Screening for viruses in all pneumonia patients may be unnecessary in clinical settings.

Our study has limitations. First, sputum samples were not available for $25 \%$ of the enrolled patients. However, the clinical characteristics of patients without sputum samples did not differ from those of patients with sputum samples. Exclusion of this group did not affect our findings. Second, we used PCR to detect viruses. The detection of viral RNA and DNA in respiratory samples does not always indicate the presence of a causal pathogen; particularly, the detection of viruses in nasopharyngeal swabs may not be reflecting lower respiratory infections in pneumonia patients. We, therefore, used sputum samples from pneumonia patients, and the presence of viruses in the lower respiratory tract must be very likely causative [31]. Third, due to the nature of an observational study design, unmeasured confounding factors may have remained in our risk factor analyses for pneumonia mortality.

\section{Conclusions}

Viral infections are common in adult pneumonia, and their impact on pneumonia mortality varies by viruses and comorbidities. Variable impacts of viruses by population characteristics must be considered in the development of antiviral drugs and vaccines. 


\section{Additional file}

Additional file 1: Table S1. Proportion of patients with multiple symptoms (number of symptoms $\geq 3$ ) by virus. Table S2. Viral and bacterial infection status and in-hospital mortality among pneumonia patients with and without aspiration risk factors. (DOCX 17 kb)

\section{Abbreviations}

ARR: Adjusted risk ratio; CAP: Community-acquired pneumonia; $\mathrm{Cl}$ : Confidence intervals; COPD: Chronic obstructive pulmonary disease; HAdV: Human adenovirus; HBoV: Human bocavirus; HCoV: Human coronavirus; hMPV: Human metapneumovirus; HPIV: Human parainfluenza virus; HRV: Human rhinovirus; PCR: Polymerase chain reaction; PS: Performance Status; RSV: Respiratory syncytial virus

\section{Acknowledgements}

We are grateful to all the laboratory staff at the participating hospitals. We would like to thank Rina Shiramizu (Institute of Tropical Medicine, Nagasaki University, Nagasaki, Japan) and Kyoko Uchibori (Institute of Tropical Medicine, Nagasaki University, Nagasaki, Japan) for performing PCR and Yumi Araki (Institute of Tropical Medicine, Nagasaki University, Nagasaki, Japan) for administrative work. Adult Pneumonia Study Group_-Japan (APSG-J) are: Takao Wakabayashi ${ }^{1}$, Naoto Hosokawa ${ }^{2}$, Norihiro Kaneko ${ }^{3}$, Kei Nakashima ${ }^{3}$ Yoshihito Otsuka ${ }^{4}$, Eiichiro Sando ${ }^{5}$, Kaori Shibui ${ }^{5}$, Daisuke Suzuki ${ }^{2}$, Kenzo Tanaka ${ }^{6}$, Kentaro Tochitani ${ }^{2}$, Masayuki Chikamori ${ }^{7}$, Masayuki Ishida ${ }^{7}$, Hiroshi Nakaoka', Hiroyuki Ito ${ }^{8}$, Kei Matsuki ${ }^{8}$, Yoshiko Tsuchihashi ${ }^{8}$, Bhim G Dhoubhadel $^{9}$, Akitsugu Furumoto ${ }^{9}$, Sugihiro Hamaguchi ${ }^{1,9}$, Shungo Katoh ${ }^{1,9}$, Satoshi Kakiuchi ${ }^{9}$, Emi Kitashoji ${ }^{9}$, Takaharu Shimazaki ${ }^{9}$, Masahiro Takaki ${ }^{9}$, Kiwao Watanabe ${ }^{9}$, Lay-Myint Yoshida ${ }^{10}$

1. Department of General Internal Medicine, Ebetsu City Hospital, Hokkaido, Japan.

2. Department of Infectious Diseases, Kameda Medical Center, Chiba, Japan. 3. Department of Pulmonology, Kameda Medical Center, Chiba, Japan. 4. Department of Laboratory Medicine, Kameda Medical Center, Chiba, Japan. 5. Department of General Internal Medicine, Kameda Medical Center, Chiba, Japan

6. Emergency and Trauma Center, Kameda Medical Center, Chiba, Japan. 7. Department of Internal Medicine, Chikamori Hospital, Kochi, Japan. 8. Department of Internal Medicine, Juzenkai Hospital, Nagasaki, Japan. 9. Department of Clinical Medicine, Institute of Tropical Medicine, Nagasaki University, Nagasaki, Japan.

10. Department of Pediatric Infectious Diseases, Institute of Tropical Medicine, Nagasaki University, Nagasaki, Japan.

\section{Funding}

This study was supported by Nagasaki University and Pfizer Japan Inc. The authors were independent from the sponsors.

\section{Availability of data and materials}

The datasets used during the current study are available from the corresponding author on reasonable request.

\section{Authors' contributions}

This study was conceived by NK, MS, MAO, KM, and KA. MAB, MY, TI, NA, NH, and MAO collected data. NK, MS, and KM performed data analysis and wrote the manuscript. All authors approved the final manuscript.

\section{Ethics approval and consent to participate}

This study was approved by the institutional review boards of the Institute of Tropical Medicine, Nagasaki University, Ebetsu City Hospital, Kameda Medical Center, Chikamori Hospital, and Juzenkai Hospital. The requirement for obtaining written consent from all participants was waived by all IRBS because of the study's observational nature without any deviation from the current medical practice.

\section{Consent for publication}

Not applicable

\section{Competing interests}

The authors declare that they have no competing interests.

\section{Publisher's Note}

Springer Nature remains neutral with regard to jurisdictional claims in published maps and institutional affiliations.

\section{Author details}

${ }^{1}$ Department of Pulmonology, Kameda Medical Center, 929 Higashi-cho, Kamogawa, Chiba, Japan. ${ }^{2}$ Division of Respiratory Medicine, Department of Internal Medicine, Kobe University Graduate School of Medicine, 7-5-1 Kusunoki-cho, Chuo-ku, Kobe, Japan. ${ }^{3}$ Department of Clinical Medicine, Institute of Tropical Medicine, Nagasaki University, 1-12-4 Sakamoto, Nagasaki 852-8523, Japan. ${ }^{4}$ Department of General Internal Medicine, Kameda Medical Center, 929 Higashi-cho, Kamogawa, Chiba, Japan. ${ }^{5}$ Department of Internal Medicine, Juzenkai Hospital, 7-18 Kagomachi, Nagasaki, Japan. ${ }^{6}$ Department of Internal Medicine, Chikamori Hospital, 1-1-16 Okawasuji, Kochi, Japan. ${ }^{7}$ Department of General Internal Medicine, Ebetsu City Hospital, 6 Wakakusacho, Ebetsu, Hokkaido, Japan.

Received: 15 August 2017 Accepted: 26 November 2017

Published online: 06 December 2017

\section{References}

1. Johansson N, Kalin M, Tiveljung-Lindell A, Giske CG, Hedlund J. Etiology of community-acquired pneumonia: increased microbiological yield with new diagnostic methods. Clin Infect Dis. 2010:50:202-9.

2. Jain S, Self WH, Wunderink RG, Fakhran S, Balk R, Bramley AM, et al. Community-acquired pneumonia requiring hospitalization among U.S. adults. N Engl J Med. 2015;373:415-27.

3. Holter JC, Muller F, Bjorang O, Samdal HH, Marthinsen JB, Jenum PA, et al. Etiology of community-acquired pneumonia and diagnostic yields of microbiological methods: a 3-year prospective study in Norway. BMC Infect Dis. 2015:15:64.

4. Burk M, El-Kersh K, Saad M, Wiemken T, Ramirez J, Cavallazzi R. Viral infection in community-acquired pneumonia: a systematic review and meta-analysis. Eur Respir Rev. 2016;25:178-88.

5. Berry $M$, Gamieldien J, Fielding BC. Identification of new respiratory viruses in the new millennium. Viruses. 2015;7:996-1019.

6. Zumla A, Hui DS, Perlman S. Middle East respiratory syndrome. Lancet. 2015; 386:995-1007.

7. Ma HM, Tang WH, Woo J. Predictors of in-hospital mortality of older patients admitted for community-acquired pneumonia. Age Ageing. 2011; 40:736-41.

8. Komiya K, Rubin BK, Kadota Jl, Mukae H, Akaba T, Moro H, et al. Prognostic implications of aspiration pneumonia in patients with community acquired pneumonia: a systematic review with meta-analysis. Sci Rep. 2016:6:38097.

9. Morimoto K, Suzuki M, Ishifuji T, Yaegashi M, Asoh N, Hamashige N, et al. The burden and etiology of community-onset pneumonia in the aging Japanese population: a multicenter prospective study. PLoS One. 2015:10:e0122247.

10. Yoshida LM, Suzuki M, Yamamoto T, Nguyen HA, Nguyen CD, Nguyen AT, et al. Viral pathogens associated with acute respiratory infections in central vietnamese children. Pediatr Infect Dis J. 2010;29:75-7.

11. Vu HT, Yoshida LM, Suzuki M, Nguyen HA, Nguyen CD, Nguyen AT, et al. Association between nasopharyngeal load of Streptococcus Pneumoniae, viral coinfection, and radiologically confirmed pneumonia in Vietnamese children. Pediatr Infect Dis J. 2011:30:11-8.

12. Oken MM, Creech RH, Tormey DC, Horton J, Davis TE, McFadden ET, et al. Toxicity and response criteria of the eastern cooperative oncology group. Am J Clin Oncol. 1982;5:649-55.

13. Lim WS, van der Eerden MM, Laing R, Boersma WG, Karalus N, Town Gl, et al. Defining community acquired pneumonia severity on presentation to hospital: an international derivation and validation study. Thorax 2003;58:377-82.

14. Reza Shariatzadeh M, Huang JQ, Marrie TJ. Differences in the features of aspiration pneumonia according to site of acquisition: community or continuing care facility. J Am Geriatr Soc. 2006;54:296-302.

15. Gadsby NJ, Russell CD, McHugh MP, Mark H, Conway Morris A, Laurenson IF, et al. Comprehensive molecular testing for respiratory pathogens in community-acquired pneumonia. Clin Infect Dis. 2016:62:817-23.

16. JX Q, Gu L, ZH P, XM Y, Liu YM, Li R, et al. Viral etiology of communityacquired pneumonia among adolescents and adults with mild or moderate severity and its relation to age and severity. BMC Infect Dis. 2015;15:89. 
17. Bewick T, Myles P, Greenwood S. Nguyen-van-tam JS, Brett SJ, Semple MG, et al. clinical and laboratory features distinguishing pandemic H1N1 influenza-related pneumonia from interpandemic community-acquired pneumonia in adults. Thorax. 2011:66:247-52.

18. Sohn CH, Ryoo SM, Yoon JY, Seo DW, Lim KS, Kim SH, et al. Comparison of clinical features and outcomes of hospitalized adult patients with novel influenza a (H1N1) pneumonia and other pneumonia. Acad Emerg Med. 2013;20:46-53.

19. Loubet P, Samih-Lenzi N, Galtier F, Vanhems P, Loulergue P, Duval X, et al. Factors associated with poor outcomes among adults hospitalized for influenza in France: a three-year prospective multicenter study. J Clin Virol. 2016;79:68-73.

20. Garg S, Jain S, Dawood FS, Jhung M, Perez A, D'Mello T, et al. Pneumonia among adults hospitalized with laboratory-confirmed seasonal influenza virus infection-United States, 2005-2008. BMC Infect Dis. 2015:15:369.

21. Hsu AC, Starkey MR, Hanish I, Parsons K, Haw TJ, Howland LJ, et al. Targeting PI3K-p110alpha suppresses influenza virus infection in chronic obstructive pulmonary disease. Am J Respir Crit Care Med. 2015;191:1012-23.

22. Poole PJ, Chacko E, Wood-Baker RW, Cates CJ. Influenza vaccine for patients with chronic obstructive pulmonary disease. Cochrane Database Syst Rev. 2006;(1):CD002733.

23. Falsey AR, McElhaney JE, Beran J, van Essen GA, Duval X, Esen M, et al. Respiratory syncytial virus and other respiratory viral infections in older adults with moderate to severe influenza-like illness. J Infect Dis. 2014; 209:1873-81.

24. Sundaram ME, Meece JK, Sifakis F, Gasser RA Jr, Belongia EA. Medically attended respiratory syncytial virus infections in adults aged $>/=50$ years: clinical characteristics and outcomes. Clin Infect Dis. 2014;58:342-9.

25. Lee N, Lui GC, Wong KT, Li TC, Tse EC, Chan JY, et al. High morbidity and mortality in adults hospitalized for respiratory syncytial virus infections. Clin Infect Dis. 2013;57:1069-77.

26. Ruuskanen O, Lahti E, Jennings LC, Murdoch DR. Viral pneumonia. Lancet. 2011:377:1264-75.

27. Goka EA, Vallely PJ, Mutton K, Klapper PE. Single and multiple respiratory virus infections and severity of respiratory disease: a systematic review. Paediatr Respir Rev. 2014;15:363-70.

28. Asner SA, Science ME, Tran D, Smieja M, Merglen A, Mertz D. Clinical disease severity of respiratory viral co-infection versus single viral infection: a systematic review and meta-analysis. PLoS One. 2014:9:e99392.

29. Jennings LC, Anderson TP, Beynon KA, Chua A, Laing RT, Werno AM, et al. Incidence and characteristics of viral community-acquired pneumonia in adults. Thorax. 2008:63:42-8.

30. Takaki M, Nakama T, Ishida M, Morimoto H, Nagasaki Y, Shiramizu R, et al. High incidence of community-acquired pneumonia among rapidly aging population in Japan: a prospective hospital-based surveillance. Jpn J Infect Dis. 2014;67:269-75.

31. Falsey AR, Formica MA, Walsh EE. Yield of sputum for viral detection by reverse transcriptase PCR in adults hospitalized with respiratory illness. J Clin Microbiol. 2012;50:21-4.

\section{Submit your next manuscript to BioMed Central and we will help you at every step:}

- We accept pre-submission inquiries

- Our selector tool helps you to find the most relevant journal

- We provide round the clock customer support

- Convenient online submission

- Thorough peer review

- Inclusion in PubMed and all major indexing services

- Maximum visibility for your research

Submit your manuscript at www.biomedcentral.com/submit

) Biomed Central 